\title{
MEJORA DE LA INTEROPERABILIDAD SEMÁNTICA PARA LA REUTILIZACIÓN DE CONTENIDOS MEDIANTE SISTEMAS DE ORGANIZACIÓN DEL CONOCIMIENTO
}

\author{
José Antonio Moreiro González ${ }^{i}$ \\ Sonia Sánchez Cuadrado ${ }^{\text {ii }}$ \\ Jorge Morato Lara ${ }^{\text {ii }}$
}

\begin{abstract}
Resumen: Los Sistemas de Organización del Conocimiento son recursos diseñados para mejorar la interoperabilidad, gestión y recuperación del conocimiento. Conforme se incrementan los recursos web se evidencia la falta de sistemas de organización del conocimiento, con el consiguiente impacto en la interoperabilidad de recursos. Los sistemas de organización del conocimiento son por definición herramientas complicadas y costosas, tanto en su creación como en su gestión. La reutilización de estructuras organizativas similares es un elemento necesario en este contexto. Se analizan experiencias de reutilización de sistemas de organización del conocimiento y se señala como los nuevos estándares están incidiendo en este aspecto.
\end{abstract}

Palabras-clave: Sistemas de Organización del Conocimiento. Interoperabilidad Semántica. Taxonomías. Ontologías.

\section{IMPROVED SEMANTIC INTEROPERABILITY FOR CONTENT REUSE THROUGH KNOWLEDGE ORGANIZATION SYSTEMS}

\begin{abstract}
The Knowledge Organization Systems (KOS) are resources designed to improve the knowledge interoperability, management and retrieval. As increases the web resources, it's evidenced the lack of KOS, with the consequent impact in the resources interoperability. The KOSS are, by definition, complicated and costly tools, so much in his creation as in his management. The reuse of similar organizational structures is a necessary element in this context. They analyses experiences of reuse of The KOS and signals like the new standards are impinged on this appearance.
\end{abstract}

Keywords: Knowledge Organization Systems. Semantic Interoperability. Taxonomies. Ontologies.

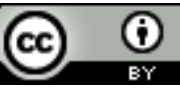

Esta obra está licenciada sob uma Licença Creative Commons

\footnotetext{
${ }^{\mathrm{i}}$ Universidad Carlos III de Madrid. jamore@bib.uc3m.es.

ii Universidad Carlos III de Madrid. sscuadra@bib.uc3m.es.

iii Universidad Carlos III de Madrid. jorge@ie.inf.uc3m.es.

Recebido em: 26/12/2011; aceito para publicação em: 12/02/2012.
} 


\section{INTRODUCCIÓN}

La recuperación de información en la Web está muy mediatizada por la sobrecarga de información y la ineficacia de las palabras-clave a la hora de representar los conceptos con pertinencia. Esto se debe a la heterogeneidad de los contenidos y de las necesidades de los usuarios que interaccionan con Internet condicionan los resultados en las búsquedas web. El problema se agrava por la carencia de una autoridad, reconocida y consensuada de forma mayoritaria, que desempeñe las funciones de control de vocabulario, reputación y fiabilidad de los contenidos. Un control de vocabulario adecuado debe justificar la existencia de los términos y sus relaciones semánticas, así como analizar y extraer nuevos términos de las fuentes publicadas. Por otro lado, la carencia de un aval de la reputación de los recursos y sus contenidos, bien sea mediante una autoridad central, bien sea mediante sistemas inteligentes, dificulta sobremanera la gestión apropiada de la información.

La reutilización se logra con la mejora de la interoperabilidad, esto es la posibilidad de que distintos recursos y aplicaciones web intercambien información a través de conceptualizaciones compartidas y una codificación común, por ejemplo, con RDF. Los conceptos, su expresión léxica, sus relaciones semánticas y las propiedades lógicas asociadas se expresan mediante Sistemas de Organización del Conocimiento con diferentes grados de conceptualización y estructuras semánticas (SÁNCHEZ-CUADRADO, 2007). Estos sistemas también son denominados KOS, por su acrónimo en inglés, Knowledge Organization Systems. La creación y desarrollo de los KOS es una tarea ardua, tanto intelectualmente como en los tiempos necesarios para su desarrollo. Sin embargo dado que la interoperabilidad depende en gran medida de las correspondencias entre distintos KOS su desarrollo es crítico. A la hora de buscar un término correspondiente entre dos o más KOS se parte de un KOS fuente (source) y se alcanza un KOS de destino (target) (BSI GROUP, 2007). Este proceso, de forma idónea, debería realizarse sin pérdida alguna de significado, de manera que el conocimiento inicialmente dispuesto para un contexto puede aplicarse en otro distinto (NOY; McGUINNESS, 2000). No obstante, cualquier conversión implica una potencial pérdida de información, y por tanto en el proceso deben aplicarse mecanismos para minimizarla.

Dado el coste de los KOS, y la necesidad que se requiere de estos recursos, las metodologías para su reutilización son un aspecto crítico en la investigación. Así, emplear el conocimiento fijado en KOS preexistentes ha alcanzado gran importancia para investigadores, documentalistas y usuarios avanzados de información a la hora de afrontar la construcción de 
nuevos lenguajes. La reutilización se vuelve obligatoria si nuestro sistema coincide, dentro de su dominio y propósitos, con el uso fiable previo de alguna ontología o de algún vocabulario controlado.

El objetivo general de este trabajo consiste en analizar las condiciones de interoperabilidad y reutilización de los KOS. Teniendo como objetivo más concreto establecer las condiciones de similitud semántica entre los preexistentes KOS de origen y los nuevos KOS de destino.

\section{INTEROPERABILIDAD Y REUTILIZACIÓN}

La interoperabilidad es la capacidad de compartir datos y posibilitar el intercambio de información de los sistemas de información y los procedimientos a los que estos dan soporte a nivel de interoperabilidad técnica, semántica y organizativa. La reutilización es una consecuencia directa ante la necesidad y los beneficios de: compartir, recuperar extraer y catalogar información univoca y pertinente.

En el ámbito de la Web 2.0, la reutilización es un proceso asociado comúnmente con aplicaciones web diseñadas para facilitar la colaboración y participación en la información, así como la interoperabilidad y el diseño centrado en el usuario (O'REILLY, 2007). La Web 2.0 da la oportunidad a usuarios, empresas e instituciones de aprovechar e integrar servicios proporcionados por otros, de manera que las entidades que participan en la Web 2.0 usan métodos incluyentes a la hora de agregar datos. Un usuario puede añadir y compartir información con otros usuarios, de forma que se genera valor añadido como efecto colateral del uso ordinario de la aplicación. Podemos afirmar, entonces, que la Web 2.0 es propensa a la reutilización, ya que apenas se aprecian barreras para hacerlo, o cuando existen son muy bajas. Sin duda actuar con alta interoperabilidad para poder intercambiar información con cualquier repositorio supone un gran ahorro económico y temporal, y menor esfuerzo para quienes se implican en su desarrollo (HUMMEL; ATKINSON, 2006).

Esta característica no es exclusiva de la Web 2.0, pues en su caracterización semántica requiere contar con sistemas de representación del conocimiento basados en ontologías para localizar y acceder a los recursos (EUZENAT; TRONCY, 2004). A la hora de desarrollar esas ontologías se pueden aprovechar organizaciones conceptuales preexistentes, por ejemplo, de 
tesauros, a lo que están dispuestas metodologías como Methontology, entre otras (GÓMEZPÉREZ; FERNÁNDEZ-LÓPEZ; CORCHO, 2004; SÁNCHEZ-CUADRADO, 2007).

El hecho de que muchos lenguajes, taxonomías y ontologías estén disponibles en formato electrónico hace que sea muy fácil importarlos a nuestro sistema. SKOS ofrece un esquema estándar para codificar vocabularios controlados y lenguajes de indización en XML. Se plantea como un método asequible para migrar al entorno de la web semántica lenguajes de indización disponibles. No debe considerarse útil solo para publicar lenguajes de indización, sino como un mecanismo para representar relaciones entre distintos esquemas conceptuales.

En la recomendación del W3C también se señala la necesidad de aprovechar la experiencia de la comunidad bibliotecaria en la organización del conocimiento, dentro de las iniciativas para la web semántica (MILES; BECHHOFER, 2009). No se busca únicamente recuperar, volcar o centralizar contenidos, sino la capacidad de relacionarlos a partir de su similitud semántica y de las relaciones reales que existen entre las entidades que participaron en su creación y gestión. En estas condiciones, reutilizar es un modo de compartir información para mejorar su representación en la Web.

\section{ANÁLISIS DE LAS CONDICIONES DE INTEROPERABILIDAD ENTRE SISTEMAS DE ORGANIZACIÓN DEL CONOCIMIENTO}

Es indudable la necesidad de interoperabilidad a la hora de elaborar o de utilizar vocabularios controlados, tanto para acceder a la información precisada como para representar el contenido específico de un campo. De manera más matizada, le incumbe a la interoperabilidad hacer eficaces la conceptualización de un recurso en otro, o tomar conceptos, y su expresión léxica, de un recurso para utilizarlo con éxito en otro. En especial, busca conjuntar varios recursos en otro mayor de cara a lograr unos mapas conceptuales y terminológicos más comprensibles.

Las normas BS 8723 (BSI GROUP, 2007), 2005-2007 y ANSI/NISO Z39.19 (2005) definen la interoperabilidad como la capacidad de dos o más sistemas o componentes de intercambiar información y de usar esa información que se ha intercambiado. Debemos pues entender que interoperabilidad es sinónimo de reutilización terminológica y esquemática. 
La demanda de interoperabilidad entre KOS se origina tanto en la tecnología de la WWW que propicia el intercambio de información en dimensiones hasta hace poco ni presentidas, como en la posibilidad de reutilización de aplicaciones y aprovechamiento de los recursos de información que fueron elaborados originalmente para un servicio y uso concretos. Esta forma de actuación se determina desde la propia aspiración de globalidad que tienen los KOS, junto a la debida accesibilidad social de la información en el sector público, así como desde la existencia de repertorios indizados mediante el empleo de algún KOS (BSI GROUP, 2007).

La interoperabilidad entre distintos KOS tiene su eje en las relaciones de equivalencia, pues antes de establecer jerarquías y asociaciones hay que contar con las equivalencias entre términos. Y esto tanto aceptando que un concepto puede representarse por dos términos preferentes distintos en dos KOS distintos de una misma lengua, como el caso de las equivalencias entre lenguas distintas, en especial de las inexactas y parciales (UNE 50125: 1997).

La norma estadounidense describe los siguientes métodos para lograr la interoperabilidad entre KOS (ANSI/NISO, 2005):

- Derivación o modelado: se selecciona un KOS fuente para desarrollar o derivar otro KOS final.

- Traducción o adaptación: algunos KOS de destino se han creado desde la traducción o adaptación de los términos de un KOS fuente en otra lengua.

- KOS satélites: el KOS fuente funciona como macroestructura para KOS más específicos (microtesauros).

- Nodo: diferentes nodos de la estructura jerárquica de un KOS fuente se vinculan a términos de un KOS más específico que se aplican a una única clase de la jerarquía.

- Mapeo directo: se establecen equivalencias y otras relaciones entre los términos de distintos KOS.

- Mapeo por coocurrencia: se busca la coocurrencia de términos en varias bases de datos para encontrar familias de términos. Los términos se seleccionan de los metadatos (palabras claves o términos controlados), o bien del texto completo.

- Conexión: un KOS de conexión contiene los puntos focales a través de los cuales se agrupan los términos de varios KOS.

- Vinculación a través de una lista de temporal unificada: diferentes KOS se vinculan mediante mapeo de términos tanto equivalentes como relacionados. El mapeo responde a la 
consulta concreta de un usuario. El resultado se muestra de forma temporal, no se genera un nuevo KOS.

- Vinculación a través de servidores de KOS: las consultas de los usuarios se envían al servidor central que pasa la consulta a los KOS registrados, recoge los distintos resultados y los presenta al usuario.

Mientras que la norma británica los limita a tres modelos básicos (BSI GROUP, 2007):

1) Unidad estructural: los KOS implicados comparten la estructura jerárquica y las relaciones asociativas entre conceptos. Se corresponde con los métodos de derivación y traducción de la norma ANSI/NISO (2005);

2) Pares no equivalentes: vincula dos KOS que no participan de la misma estructura, pero que resultan de combinar taxonomías, tesauros, encabezamientos de materia, listas de autoridades, etc. Se identifica con el mapeo directo de la norma ANSI/NISO (2005);

3) Estructura básica: la complejidad que supone vincular tres o más KOS obliga a seleccionar uno de los KOS como estructura básica. Es igual que el método de conexión de la norma ANSI/NISO (2005).

\section{FACTORES QUE AFECTAN A LA INTEROPERABILIDAD}

Se derivan con facilidad los problemas que pueden darse cuando se utiliza un KOS en diferentes dominios o sistemas, o cuando se fusionan dos o más KOS para formar un lenguaje nuevo que contendrá los conceptos y términos de los originales. Su éxito está condicionado por estos factores (ANSI/NISO, 2005):

1) La semejanza de los conceptos de una materia incluida en dominios diferentes. De especial relevancia cuando se emplea un KOS único para indizar o buscar en recursos de contenido heterogéneo;

2) Uso de diferentes KOS para indizar contenidos de dominios similares. Se da esta situación cuando se emplean diferentes KOS para representar el contenido de uno o más dominios. De manera que pueden emplearse dos o más KOS distintos para indizar los mismos textos especializados. De manera que los términos incluidos en diferentes KOS pueden variar su expresión, especificidad y jerarquización -incluso respetando el estándar-, haciendo que los resultados recuperados sean imprevisibles en dominios diferentes; 
3) El grado de especificidad o granularidad de los lenguajes documentales empleados para indizar diferentes dominios o bases de datos. Debido a las diferencias de especificidad de los términos empleados para indizar, si se usa un término más granular de un KOS puede no recogerse los resultados deseados en otra base de datos donde la indización se hizo de forma más genérica para representar el mismo concepto;

4) Las metodologías esperadas para realizar búsquedas en bases de datos. Las bibliotecas han manejado este problema mediante el uso de Z39.50, un protocolo de búsqueda común para realizar búsquedas a través de OPACs. Incluso así, con un protocolo tan común, la interpretación de términos de búsqueda y los resultados logrados podrían ser muy diferentes dependiendo de si una base de datos espera una búsqueda precoordinada o una postcoordinada. Por ejemplo, un KOS podría haber sido desarrollado para asistir a la navegación en Web mientras otro se construyó para proporcionar términos para consultas postcoordinadas mediante operadores booleanos;

5) Las garantías literaria y organizativa empleadas en el desarrollo del vocabulario. Si las garantías de cada sistema o base de datos son bastante diferentes, podría darse una equivalencia muy parcial entre términos de diferentes vocabularios o un mismo término podría tener significados diferentes sin distinguir su calificador;

6) La finalidad buscada por las bases de datos. Cuando los propósitos o las posibles audiencias son muy variados, los usuarios podrían verse decepcionados con los resultados de sus búsquedas. Por ejemplo, los resultados obtenidos en una base de datos de ayudas al estudio para estudiantes menores de 12 años serían muy diferentes de una búsqueda de alta exigencia en una base de datos científico-técnica como PubMed.

\section{REUTILIZACIÓN DE TAXONOMÍAS Y DE ONTOLOGÍAS}

Desde hace décadas, los dominios científicos gestionan su información con lenguajes controlados. Muchos de ellos han ido evolucionando hacía estructuras con una semántica más rica, adquiriendo las ontologías una creciente relevancia. El primer paso a dar por quien quiera crear una taxonomía o una ontología es el de saber si existen taxonomías, tesauros u ontologías sobre ese mismo asunto o en su proximidad. Para ahorrar tiempo y esfuerzo valen incluso listas de términos bien conformadas. Entre los objetivos que persiguen las taxonomías y ontologías se destaca el de aprovechar la reutilización del conocimiento perteneciente a un 
dominio (NOY; McGUINNES, 2000). Así pues, es una condición inherente de las ontologías que el conocimiento al que atienden sea reutilizable. La reutilización se hace obligatoria cuando un sistema coincide, dentro de un dominio y propósitos concretos, con el uso fiable previo de alguna ontología o de algún vocabulario controlado. Nuestra información vendría a ampliarlos y refinarlos.

Precisamente una de las ventajas que ofrecen las ontologías descansa en la posibilidad de reutilizar toda la estructura clasificatoria o parte del desarrollo jerárquico de las clases desde ontologías preexistentes con la finalidad de adaptarlas a la que se está elaborando, incluso en sistemas heterogéneos (QIN; PALING, 2001). Aunque cada ontología responde a un modo diferente de entender un estado determinado del conocimiento, siempre es posible utilizar algunos elementos de otras anteriores, de acuerdo con las necesidades. De manera que, con frecuencia, se captura la taxonomía principal, o se emplea la ontología de origen como base terminológica para la ontología de destino, evitando trasladar otras propiedades, axiomas o instancias (SABOU et al., 2006). Viene facilitado porque muchas ontologías están disponibles para poderlas importar. Tanto en la web, como en la bibliografía especializada existen repositorios de ontologías reutilizables:

- DAML (http://www.daml.org/ontologies/), UNSPSC (www.unspsc.org), RosettaNet (www.rosettanet.org), DMOZ (www.dmoz.org), KIF (GENESERETH; FIKES, 1992);

- Combinando marcos con lógica de primer orden: Ontolingua (http://www.ksl.stanford.edu/software/ontolingua/), CycL (LENAT; GUHA, 1990), OCML (MOTTA, 1999) y Flogic (KIFER; LAUSEN; WU, 1995);

- Finalmente LOOM (MACGREGOR, 1991) con lógica descriptiva.

Según Sánchez y Gil, "Las diferencias entre lenguajes documentales y ontologías complican el traslado del conocimiento existente en un tesauro o un sistema de clasificación a una ontología" (SÁNCHEZ JIMÉNEZ; GIL URDICIAIN, 2007).

En los últimos años se han abordado muchos proyectos de interoperabilidad entre KOS, que pueden clasificarse así (MARTÍNEZ TAMAYO et al., 2010):

- Entre listas de encabezamientos de materia: un ejemplo es el proyecto MACS de la Conference of European National Libraries (CENL), que ha vinculado tres listas de epígrafes: LCSH en inglés, Répertoire d'autorité-matière encyclopédique et alphabétique unifié (Rameau) en francés y Schlagwortnormdatei (SWD) en alemán (LANDRY, 2000);

- Entre tesauros (postcoordinados): entre ellos el proyecto ASESG del Centro de Ecosistemas Acuáticos de Cataluña, que ha vinculado cinco tesauros que abarcan las ciencias 
acuáticas, pesca, agricultura, alimentación, medio ambiente y economía (REVERTÉ; SEBASTIÁ, 2009);

- Entre listas de encabezamientos de materia y tesauros: el proyecto SWD-IZ es una experiencia alemana que relaciona los conceptos indizados en dos bases de datos, una que utiliza el Thesaurus Sozialwissenschaften (IZ) de ciencias sociales y la otra que usa la lista de epígrafes SWD (ZHANG, 2006);

- Entre sistemas de clasificación: Library of Congress ha establecido equivalencias entre las notaciones de Library of Congress Classification (LCC) y las del Sistema de Clasificación Decimal de Dewey (SCDD) (LIBRARY OF CONGRESS, 2004);

- Entre listas de epígrafes, tesauros y sistemas de clasificación: un ejemplo es el proyecto KoMoHe del Bundesministerium für Bildung und Forschung de Alemania, iniciado en 2004 y concluido en 2007. En total se han vinculado 25 SOC (16 tesauros, 4 listas de descriptores, 2 listas de encabezamientos de materia y 3 sistemas de clasificación, incluyendo SCDD), de 11 disciplinas, en alemán, inglés y ruso (MAYR; PETRAS, 2009);

- Fusión de ontologías. La ontología relacional OBO (Open Biomedical Ontologies) fue diseñada para evitar inconsistencias en la relación conceptual y mejorar la interoperabilidad de los distintos recursos del sector biomédico (SMITH et al., 2005);

- Representación común de esquemas de metadatos. El proyecto SEmantic Metadata SEarch (SEMSE) propone un plano semántico para la representación de esquemas de metadatos de forma que se posibilite su recuperación conceptual basada en el significado y contenido de sus elementos (PALACIOS et al., 2010; LLORENS; MORATO, 2010).

La reutilización puede entenderse como un proceso que empareja el auténtico significado tanto de la necesidad de información como de las descripciones del producto destinado a resolverla. Se trata de mapear los términos de una ontología con los de otra a través de una serie de preguntas y respuestas. Se modelan las prácticas existentes en un dominio para lo que reutilizan los procesos de negociación. Sin este proceso de negociación, las ontologías proporcionan poco valor para la reutilización más allá de la práctica en modelización de dominios (BAILIN, 2004). El proceso se hace como si sucediese en una aplicación de espacio-sistema en el que los usuarios finales "programan" funciones nuevas integrando componentes reutilizables.

Incluso existen ensayos que se inclinan por interpretar las ontologías como un recurso léxico, tal que incluyen tesauros (SÁNCHEZ-CUADRADO, 2007). Así, al evaluarse el comportamiento en fusión automática de las ontologías de alto nivel SUMO, DOLCE y 
OpenCyc, que se comparan desde la probabilidad de encontrar cadenas de palabras similares (MASCARDI; LOCORO; ROSSO, 2009).

Dentro de las distintas experiencias parecen tener una gran importancia los tesauros. Se consideran KOS ligeros con una dilatada trayectoria en la gestión de información y en los primeros estadios de la creación de KOS complejos, como ya se ha comentado. Los KOS dado su mayor simplicidad estructural y semántica son un elemento clave para la reutilización. Este parece ser el principio rector que ha llevado a una actualización de las normas sobre el tema en lo relativo a la interoperabilidad. Ejemplos son: ANSI 39.50 de 2005, revisada en 2010, dando un mayor énfasis a la interoperabilidad. La británica BS8723 de los años 2010 y 2011. En preparación, y de interés en este aspecto, está la ISO25964 que da rango internacional a la BS8723. Además está la de la IFLA 115 (2009), que actualiza algo la ISO 2788:1986 (tesauros monolingües) y 5964:1985 (tesauros multilingües).

\section{CONCLUSIONES}

Crear ontologías consensuadas es una tarea lenta y compleja. En cualquier dominio resulta difícil poner de acuerdo a los diferentes participantes para definir una ontología en común. El papel de los usuarios-autores de una ontología es fundamental en su elaboración pues la estructura y conceptos que se incluyan en la ontología depende del punto de vista del que se parta. Toda ontología refleja una concepción específica de la realidad y del dominio de interés. La diferencia de perspectiva llega a alcanzar incluso a las traducciones de los conceptos contenidos en una misma ontología, ya que los términos pueden alterar su significado al ser traducidos. Para garantizar la interoperabilidad de las ontologías habría que definir unas ontologías genéricas comunes, útiles para el intercambio de información entre distintas partes, que sean compatibles con las ontologías particulares de cada área de interés como las ontologías de alto nivel o top ontologies.

Con todo, reutilizar no es sencillo. Siempre será preciso evaluar las ontologías candidatas para reconocer cual es la que se adapta mejor a las exigencias de nuestro proyecto. Aunque siempre habrá que saber si su acceso es gratuito o no. Las diferentes relaciones semánticas y estructuras lógicas deductivas existentes en las ontologías complican el traslado del conocimiento que contienen los lenguajes documentales combinatorios y los sistemas clasificatorios. Más sencillo cuando se trata de taxonomías. Hacer explícitas las relaciones 
existentes en las ontologías requiere una atención muy detallada, pues no pueden nunca derivarse directamente de las que existen en los sistemas de organización del conocimiento precedentes.

\section{REFERÊNCIAS}

ANSI/NISO. Z39.19-2005. Guidelines for the Construction, Format, and Management of Monolingual Controlled Vocabularies. 2005. Disponíble en: $<$ http://www.niso.org/standards/index.html >. Acceso en: 08 ago. 2011.

BAILIN, S. C. Software Reuse as Ontology Negotiation. In: BOSCH, J.; KRUEGER, C. (Eds.) ICSR 2004. LNCS 3107. Heidelberg: Springer, p. 242-253, 2004.

BSI GROUP (2005-2007). Structured vocabularies for information retrieval: guide. London: BSI (BS 8723/1-4), 2007.

EUZENAT, J. Y TRONCY, R. Web sémantique et pratiques documentaires, en: Le Moal, J. C. ; Hidoine, B. y Calderan, L. (éds). Publier sur internet. Paris: ADBS, 2004. p. 157-188.

GENESERETH, M. R.; FIKES, R. E. Knowledge Interchange Format, Version 3.0. Reference Manual. Computer Science Department. Stanford University. Technical Report Logic-92-1, 1992.

GÓMEZ-PÉREZ, A.; FERNÁNDEZ-LÓPEZ, M.; CORCHO, O. Ontological Engineering: with examples from the areas of knowledge management, e-commerce and the Semantic Web. London, Springer, 2004. p. 403.

HUMMEL, O.; ATKINSON, C. Using the Web as a Reuse Repository. In: MORISIO, M. (Ed). ICSR 2006. LNCS 4039. Berlin-Heidelberg: Springer, 2006.

IFLA 115. Guidelines for Multilingual Thesauri. The Hague: IFLA, 2009 (IFLA Professional Reports 115). Disponible en: $<$ http://archive.ifla.org/VII/s29/pubs/Profrep115.pdf $>$. Acceso en: 23 nov. 2011.

KIFER, M.; LAUSEN, G.; WU, J. Logical foundations of object-oriented and frame-based languages. Journal of the ACM, v. 42, n. 4, p. 741-843, 1995.

LANDRY, P. The MACS Project: Multilingual access to subject. In: International Cataloguing and Bibliographic Control. v. 30, n. 3. p. 46-49, 2000.

LENAT, D. B.; GUHA, R. V. Building large knowledge-based systems: representation and inference in the Cyc project. Addison-Wesley: 1990.

LIBRARY OF CONGRESS. Classificaton Web: quick start tutorial. Washington DC: The Library, 2004. Disponible en: $<$ http://classificationweb.net/tutorial/1 intro.html $>$. Acceso en: 23 nov. 2011. 
LLORENS, J.; MORATO, J. SEMSE: Semantic Metadata Search: TIN 2007-67153. Jornada de Seguimiento de Proyecto 2010. Programa Nacional de Tecnologías Informáticas. España, 2010 .

MACGREGOR, R. M. Using a Description Classifier to Enhance Deductive Inference. Proceedings Seventh IEEE Conference on AI Applications. p. 141-147, 1991.

MARTÍNEZ TAMAYO, A. M. et al. La interoperabilidad entre sistemas de organización del conocimiento: un nuevo desafío. I Jornada de Intercambio y Reflexión acerca de la Investigación en Bibliotecología, La Plata, 6-7 de diciembre de 2010. La Plata: Facultad de Humanidades y Ciencias de la Educación de la Universidad Nacional de La Plata, 2010. Disponible en: <http://jornadabibliotecologia.fahce.unlp.edu.ar/jornada-2010/martinez2>. Acceso en: 08 ago. 2011.

MASCARDI, V.; LOCORO, A.; ROSSO, P. Automatic Ontology Matching Via Upper Ontologies: A Systematic Evaluation. IEEE Transactions on Knowledge and Data Engineering, p. 1-14, 2009.

MAYR, P.; PETRAS, V. Cross-concordances: terminology mapping and its effectiveness for information retrieval. En International Cataloging and Bibliographic control. v. 38, n. 3. p. 43-52, 2009.

MILES, A.; BECHHOFER, S. (Ed.). SKOS Simple Knowledge Organization System Reference. W3C Recommendation 18 August 2009. Disponible em: $<$ http://www.w3.org/TR/2009/REC-skos-reference-20090818/>. Acceso en: 24 jun. 2011.

MOTTA, E. Reusable Components for Knowledge Modelling. Netherlands: IOS Press, 1999.

NOY, N. F.; McGUINNESS, D. L. Ontology development 101: a guide to creating your first ontology. Palo Alto: Stanford University, 2000. Disponible en: $<$ http://protege.stanford.edu/ontology101-noy-mcguinness.html $>$. Acceso en: 24 jun. 2011.

O'REILlY, T. What Is Web 2.0? Design Patterns and Business Models for the Next Generation of Software. MPRA Paper, n. 4580, 2007. Disponible en: <http://mpra.ub.unimuenchen.de/4580/>. Acceso en: 09 jun. 2011.

PALACIOS, V. et al. Tagging for Improved Semantic Interpretation of XML, CIKM Workshop on Exploiting Semantic Annotations in Information Retrieval, 2010, p. 19-20.

QIN, J.; PALING, S. Converting a controlled vocabulary into an ontology: the case of GEM. Information Research, v. 6, n. 2, 2001. Disponible en: <http://informationr.net/ir/62/paper94.html>. Acceso en: 23 nov. 2011.

REVERTÉ, C.; SEBASTIÁ, M. Aquatic science subject Gateway Project as a model of interoperability. En ISKO-UK 2009 Conference. London: ISKO-UK Chapter, 2009. Disponible en: < http://www.iskouk.org/conf2009/papers/reverte_ISKOUK2009.pdf>. Acceso en: 23 nov. 2011. 
SABOU, M. et al. Ontology Selection: Ontology Evaluation on the Real Semantic Web. Workshop: Evaluation of Ontologies for the Web (EON 2006) at 15th International World Wide Web Conference. WWW 2006. Edimburg. 2006, p. 189-195.

SÁNCHEZ-CUADRADO, S. Definición de una metodología para la construcción automatizada de sistema de organización del conocimiento. Tesis Doctoral, Universidad Carlos III, abril, 2007.

SÁNCHEZ JIMÉNEZ, R.; GIL URDICIAIN, B. Lenguajes documentales y ontologías. El profesional de la información, v. 16, n. 6, p. 551-560, 2007.

SMITH, B. et al. Relations in biomedical ontologies. Genome Biology, V. 6, 2005. Disponible en: <http://genomebiology.com/2005/6/5/R46>. Acceso en: 27 jun. 2011.

UNE 50125:1997. Documentación - Directrices para la creación y desarrollo de tesauros multilingües. 1997.

ZHANG, X. Concept integration of document databases using different indexing languages. Information Processing \& Management. v. 42, n. 1, p. 121-135, 2006. 